\title{
Physicochemical Characteristics of Nanocomposites under Environmental Exposure Conditions for Space Applications
}

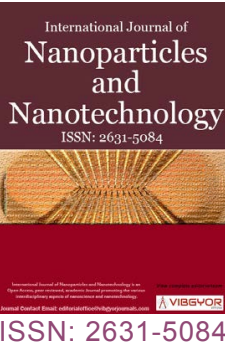

\section{Sung Hee Joo ${ }^{1 *}$, Robert S Arnold', Cecilia M Luciano ${ }^{2}$, W William Benedict ${ }^{3}$, Tyra Collins ${ }^{4}$, Yordany C Guerra ${ }^{1}$ and Kevin $R$ Luongo ${ }^{5}$}

${ }^{1}$ Department of Civil, Architectural, and Environmental Engineering, University of Miami, USA

${ }^{2}$ Chemical Engineering Department, Stanford University, USA

${ }^{3}$ Rosenstiel School of Marine and Atmospheric Science, University of Miami, USA

${ }^{4}$ Department of Biomedical Engineering, The Howard Hughes Medical Institute, University of Miami, USA

${ }^{5}$ Bionium Laboratory, Miller School of Medicine, University of Miami, USA

\begin{abstract}
Despite well-perceived advancements of nanotechnology for space applications, application of nanotechnology in the aerospace industry has potential challenges in the perspectives of monitoring and accurately measuring types and levels of pollution. Nanomaterials applied for chemical nanosensors should be well characterized and designed to detect contaminant levels different from Earth's. However, a few studies have investigated the transformation and efficacy of aged nanomaterials in sensor development. The present study explored the aging effect of oxygenation, UV irradiation, and heat treatment on selected nanomaterials (i.e., GO, CNTs, $\mathrm{ZnO}$ nanowire, $\mathrm{TiO}_{2}$, and $\mathrm{ZnO}$ ) in the perspective of sensing performance to detect trace contaminants.

Further, GO-CNT nanocomposite thin films deposited on electrode chips were exposed to the $\mathrm{CO}_{2}$ gas contaminant, and their electrical resistance was measured. Results indicate significant changes in physicochemical properties (particle size, zeta potential, and absorbance) of the model nanomaterials under oxygenation and UV irradiation, which was further investigated on their electric resistance upon exposure to gaseous contaminants. The assessment of changes to the $\mathrm{CO}_{2}$-sensing ability of carbon-nanotube or graphene-oxide hybrid thin films suggests decreasing sensitivity under both UV irradiation and oxygenation.
\end{abstract}

\section{Keywords}

Aged nanoparticles, Graphene oxide, Multi-walled carbon nanotube, Gas detection, Space applications

*Corresponding author: Sung Hee Joo, Department of Civil, Architectural, and Environmental Engineering, University of Miami, 1251 Memorial Dr. McArthur Engineering Building Coral Gables, FL 33146-0630, USA, Tel: +1-305-284-3489, Fax +1-305-284-3492

Accepted: April 30, 2019; Published: May 02, 2019

Copyright: (C) $2019 \mathrm{Joo} \mathrm{SH}$, et al. This is an open-access article distributed under the terms of the Creative Commons Attribution License, which permits unrestricted use, distribution, and reproduction in any medium, provided the original author and source are credited.

Joo et al. Int J Nanoparticles Nanotech 2019, 5:025 


\section{Introduction}

Several nanomaterials (NMs) including graphene, carbon nanotubes (CNTs), metal-oxide nanowires (NWs) have recently emerged as intriguing points of research in multidisciplinary fields, since these NMs exhibit enhanced structural, electrical, and optical properties [1-9]. Thus, they may serve as promising sources of innovation in space applications. Examples of such applications include launching equipment into space with desirably lightweight materials (high strength-to-weight ratio), in which nanotechnology plays a key role in the structure that incorporates various devices (e.g., sensors, actuators, electronic and photonic devices) $[1-3,10]$. Among these devices, particular attention is given to gas sensors, because of their crucial role in managing the hazardous risks of gas accumulation during space missions (e.g., $\mathrm{CO}, \mathrm{CO}_{2}$, methane, $\mathrm{NO}_{x^{\prime}}$ and $\mathrm{SO}_{2}$ ) $[11,12]$. Development of sensitive, reliable, and lightweight gas sensors (i.e., reducing dependent weight and increasing the structural strength of such devices) for a spacecraft is imperative to ensure accurate monitoring and safer space environments $[13,14]$.

Among NMs applied in gas sensors, graphene oxide (GO) and CNTs exhibit high selectivity and sensitivity, along with their lightweight property, when interacting with gas molecules $[1,2,9,10,15,16]$. Five model NMs chosen in the present study include $\mathrm{GO}, \mathrm{ZnO} \mathrm{NW}, \mathrm{ZnO}, \mathrm{TiO}_{2}$, and CNTs; these NMs were chosen based on several properties, which include but are not limited to high sensitivity in gas detection (i.e., graphene sensors) $[2,9,17]$, increasing electrical conductance (i.e., GO) and sensing properties at room temperature $[2,16]$, superior operation at room temperature (i.e., CNTs), and increased interaction with gas molecules due to high specific surface area of CNTs $[1,10]$. Hybrid gas sensors, which are synthesized by incorporating CNTs into metal oxide gas sensors, have been found to have improved gas-sensing capability, due to the improved surface-to-volume ratio that CNTs offer [18].

Zinc oxide, which has promising optical properties, has a wide range of applications (e.g., gas sensing, UV detection, and solar cells) in its nanowire form $[19,20]$. Additionally, $\mathrm{ZnO}$ as a gas sensor has revealed accurate monitoring and rapid response in detecting reactive gases due to its selectivity and sensitivity $[21,22]$. The versatility of $\mathrm{ZnO}$, which can be manipulated under different environments as a means of gas sensing, enables it to take various forms of nanowires, nanosheets, nanowalls $[23,24]$. Similarly, the ZnO NW sensor has been found to have great capacity for space applications due to its rapid response to UV radiation and hydrogen gas [19], and resistance of ZnO NWs higher than usual was shown when these were exposed to oxygen [25]. $\mathrm{TiO}_{2}$ has shown promise in gas applications because of its unique photovoltaic and photocatalytic properties $[26,27]$, and it has the surface composition with unique binding sites, enabling wide selectivity when employed as a gas sensor $[28,29]$. Further, $\mathrm{TiO}_{2}$ offers supporting functions for various nanostructures and enhances sensing capacity of hybrid nanosensors [30].

Despite the crucial role of nanotechnology in space devices, the long-term viability of commonly used NMs has not been thoroughly examined, and no study is found regarding the aging effect (longterm viability) of the selected NMs (i.e., CNTs, ZnO, $\mathrm{TiO}_{2}, \mathrm{GO}$, and $\mathrm{ZnO}$ nanowires) on sensitivity and selectivity under environmental conditions to detect trace levels of contaminants. The novelty of the present study lies in addressing potential issues regarding aged/transformed NMs and their sensitivity in detecting the levels of contaminants in space. In the study, environmental conditions such as oxygenation, UV irradiation, and heat treatment were chosen to examine the aging effect of the model nanomaterials (CNTs, $\mathrm{ZnO}, \mathrm{TiO}_{2}, \mathrm{GO}$, and ZnO nanowires) on sensitivity, and such effect from exposure was studied through monitoring particle size, zeta potential, and absorbance and conducting qualitative analysis of NMs through scanning electronic microscopy (SEM) and transmission electron microscopy (TEM), as well as a $\mathrm{CO}_{2}$-sensing ability study of CNT/GO hybrid thin films to examine the long-term viability of a CNT-GO sensor.

The overall goal of the present study is to assess the efficacy of the selected NMs when those are exposed to environmental conditions different from Earth's and characterize versatile NMs for their appropriateness in space products. Two hypotheses driven on this research include a) NMs may affect the strength, feasibility, longevity, effectiveness, and even accuracy in measuring/monitoring contaminants of space devices and b) A change in the resistance (ohms) of the thin films (i.e., GO-CNT) after exposure to $\mathrm{CO}_{2}$ may depend on different en- 
vironmental conditions in space. Specific objectives include 1) Examining fabrication and characterizing selected single or hybrid NMs and 2) Assessing versatile NMs for their appropriateness in space products.

\section{Materials and Methods}

\section{Materials and equipment}

The nanomaterials used were multi-walled carbon nanotubes (Sigma-Aldrich Chemistry, St. Louis, MO), single-layered graphene oxide (Advanced Chemical Supplier, Pasadena, CA), zinc-oxide nanopowder ( $<50 \mathrm{~nm}$ particle size) (Sigma-Aldrich Chemistry, St. Louis, MO), a zinc-oxide nanowire (diameter $\times$ length $=50 \mathrm{~nm} \times 300 \mathrm{~nm}$ ) (Sigma-Aldrich Chemistry, St. Louis, MO), and titanium(IV) oxide nanopowder ( $<25 \mathrm{~nm}$ particle size) (Sigma-Aldrich Chemistry, St. Louis, MO). The equipment employed include an Adventurer SL Scale (Ohaus, Parsippany, NJ); VWR Stir Plates (VWR, Radnor, PA); a $100 \mathrm{~W}, 240 \mathrm{~V}$ short-wavelength ultraviolet (UVC) lamp; a Precision Hot Water Bath (Thermo Fisher Scientific, Waltham, MA); a pH Probe (Thermo Fisher Scientific, Waltham, MA); a Malvern Zetasizer (Malvern Instruments, Malvern, United Kingdom); a Branson 1510 Sonicator Bath (Branson, Danbury, CT); a Shimadzu UV-1280 Spectrophotometer (Shimadzu Corp, Kyoto, Japan); and a VWR Mini Vortexer (VWR, Radnor, PA).

\section{Analytical procedures of samples}

A total volume of $50 \mathrm{mgL}^{-1}$ was prepared from deionized water $(100 \mathrm{~mL})$ for each of the five nanoparticle compounds studied: graphene oxide (GO), carbon nanotube (CNT), $\mathrm{TiO}_{2}, \mathrm{ZnO}$, and a $\mathrm{ZnO}$ nanowire. Four identical suspensions $\left(\mathrm{ZnO}, \mathrm{TiO}_{2}\right.$, ZnO NW, and GO), which were exposed to the three experimental conditions (heat, oxygen, and UV), and control samples were analyzed for their physicochemical properties and electric resistance. All samples exposed to environmental conditions (oxygenation, UV irradiation, and heat) were subject to 28 cumulative hours per week and during exposure periods, the experimental apparatus was contained in a low-light environment; such a setup was important to restrict extraneous light interference.

The application of exposure conditions is described as follows. Oxygen exposure conditions were achieved by exposing each of the four distinct nanoparticle suspensions to flowing air at a flow rate of $1.021 \times 10^{-4} \mathrm{~m}^{3} \mathrm{~s}^{-1}$. Heat exposure conditions were achieved by exposing suspensions to a water bath at $50{ }^{\circ} \mathrm{C}$. Ultraviolet exposure conditions were achieved by UV illumination of nanomaterial suspensions using a short-wavelength ultraviolet (UVC) lamp at a voltage of $240 \mathrm{~V}$ and a $100 \mathrm{~W}$ power. During illumination, the experimental apparatus was enclosed in an aluminum foil to apply UV light and obstruct peripheral light's entry, and the suspensions were held in 'KIMAX' flasks made of borosilicate glass.

To maintain uniformity in the nanomaterial suspensions during exposure, all suspensions were continually stirred while undergoing experimental conditions (except for heat conditions, since the samples were submerged in a water bath). The triplicate set of nanoparticle suspensions was employed as a control group. This set of suspensions remained in a dark, enclosed environment, and non-control group suspensions were also kept in this environment when not undergoing specific exposure conditions.

\section{Analysis of physicochemical properties}

Nanoparticle suspensions were exposed for 14 days (over accumulative 56 hours), and triplicate measurements were taken during each run. Particle size and zeta potential were measured using a Malvern Zetasizer from $1 \mathrm{~mL}$ aliquots of each suspension. A Thermo Scientific $\mathrm{pH}$ probe was utilized to obtain $\mathrm{pH}$ measurements from 2 $\mathrm{mL}$ aliquots of each suspension, and a Shimadzu UV-1280 Spectrophotometer was used to obtain absorbance measurements from those aliquots. For SEM preparation, each particulate sample was placed on an aluminum stub that was covered with a carbon adhesive tab, coated with a $20 \mathrm{~nm}$ thin Pd coating in a Cressington Sputter Coater, placed in an FEI XL-30 Field Emission Scanning Electron Microscope, and imaged at several magnifications. Afterwards, micrographs were saved digitally. As for the TEM analysis, each sample was diluted, and a drop of the solution was placed on a formvar/ carbon-coated 20 mesh copper grid and subjected to air drying. Afterwards, the samples were imaged at several magnifications, and a Gatan camera fitted to a Philips CM-10 TEM was used for imaging at the TEM Core Lab at the University of Miami Miller School of Medicine. 


\section{Analysis of electrical resistance of GO-CNT nanocomposite thin films}

Nanocomposite thin (GO-CNT) films were deposited on an electrode chip in a vacuum cleanroom. The substrate on the electrode chip was made of fresh and aged samples along with exposure conditions (e.g., aged GO-aged CNT, UV-exposed GOUV-exposed CNT, and oxygenated GO-oxygenated CNT). The electrode chips consisted of micro-fabricated electrodes on the surface of a single-crystal silicon wafer coated with silicon dioxide, diced 1 by $1 \mathrm{~cm}^{2}$. Before starting the deposition process, the chips were placed inside of a $50 \mathrm{~mL}$ beaker and rinsed properly with acetone, then isopropanol, and finally DI water to eliminate any polymers.

To deposit the thin film, a hot plate was turned on and set up to $100{ }^{\circ} \mathrm{C}$. Once the hot plate reached the desired temperature $\left(100{ }^{\circ} \mathrm{C}\right)$, a chip was placed on it. A $100 \mu \mathrm{L}$ pipette was used to deposit three times $30 \mu \mathrm{L}$ of solution, separately for $\mathrm{GO}$ and CNT. Each time that $30 \mu \mathrm{L}$ of solution was added on the surface of the chip, it was allowed to dry and evaporate. These steps were repeated three times, alternating for each nanomaterial and obtaining a final nanocomposite thin film made up of a total of $90 \mu \mathrm{L}$ of GO and $90 \mu \mathrm{L}$ of CNT. The same procedure was repeated for each condition to which the nanoparticles were exposed (oxygen, UV, control-aged, and control-fresh). Once the nanocomposite thin films had been deposited on a chip for each condition, samples were placed in a sealed container and taken out of the cleanroom to the regular lab to conduct the experiment.

The equipment used for these experiments was prepared before resistance measurements were taken and before and after exposure to the $\mathrm{CO}_{2}$ gas contaminant. The Printed Circuit Board (PCB) used for the purposes of these experiments consisted of two layers, $20 \mathrm{~mm}$ width and $50 \mathrm{~mm}$ length with four copper traces on opposite and alternate sides of the board. Each copper trace was approximately $6 \mathrm{~mm}$ wide and $18 \mathrm{~mm}$ long. Two spring clips were welded on alternate sides of the PCB. A Keithley 2420 source meter was turned on and adjusted to "Ohm" option (M $\Omega$ ), and cables were properly connected while making sure that there was current flow before measurements started. The chamber used for the purposes of these experiments consisted of a cubic crystal box of $21 \mathrm{~cm}^{2}, 2 \mathrm{~cm}$ thick with eight clips for hermetic seal. On its top, there was a vacuum pump for monitoring air pressure. Inside, there were four conducting cylindrical tubes used to measure any type of electric current (e.g., resistance).

The chamber was set up by connecting the $\mathrm{CO}_{2}$ tank to the vacuum pump for $\mathrm{CO}_{2}$ exposure. On finalization of the equipment set-up, the electrode chip was placed on the PCB, held by the two spring clips inside the chamber. To properly place an electrode chip onto the PCB, both spring clips touched parallel electrodes on chip at all times and in a steady manner. The two PCB clips always made contact with two of the four conducing cylindrical tubes of the chamber. Finally, using the source meter, measurements of resistance were taken for each thin film. Cables (clamps preferably) were connected from the source meter to the chamber for resistance measurements, and values were recorded.

To record resistance values after exposure, the $\mathrm{CO}_{2}$ tank was opened slightly to allow a current of $\mathrm{CO}_{2}$ to enter the chamber. Then, after 60 seconds of acclimatization, exposure to $\mathrm{CO}_{2}$ was allowed before the resistance on the electrode chip was measured. After time had elapsed, the resistance was again recorded. The same procedure was repeated for each condition to which the nanoparticles were exposed (oxygen, UV, control-aged, and control-fresh). The schematic diagram of the overall experimental procedure is shown in Scheme 1. Statistical significance was confirmed and determined using the Holm-Sidak method, with alpha = 0.05. Each row was analyzed individually, without assuming a consistent SD.

\section{Results and Discussion}

\section{Physicochemical properties of the model nanomaterials under environmental exposure}

As one of the physicochemical properties of the model NMs, absorbance was measured when the NMs were exposed to each environmental condition (i.e., aging, oxygenation, UV irradiation, and temperature). Since absorbance is the ability of a material to absorb light [31], the absorbance value of the material would be low when a large amount of light was capable of passing through a material. By contrast, when a small amount of light passed through the material, the absorbance value of the material would be high. 
As shown in Figure $1 \mathrm{~A}$, statistically declining trends for oxygenation, heat, and UV exposure were observed from the NMs, except GO. GO may not undergo transformation under the environmental conditions. This result is consistent with a study [32] in which GO showed no degradation when GO was exposed to a total of 700 hours of UV-A radiation and humidity at $50^{\circ} \mathrm{C}$, elucidating a similar absorbance value of $\mathrm{GO}$ after 700 hours of exposure to environmental conditions. This suggests UV radiation may not have a detrimental effect on GO. Notably, ZnO NW showed a significantly declining trend over all environmental conditions, and $\mathrm{ZnO}$ exhibited significant absorbance decrease under $\mathrm{O}_{2}$
A)

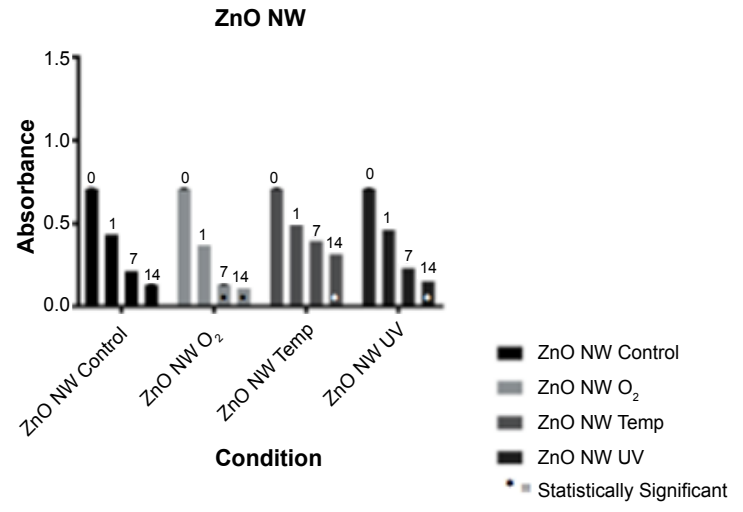

$\mathrm{TiO}_{2}$

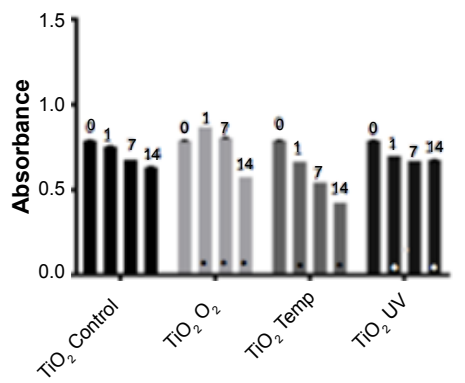

Condition

B)

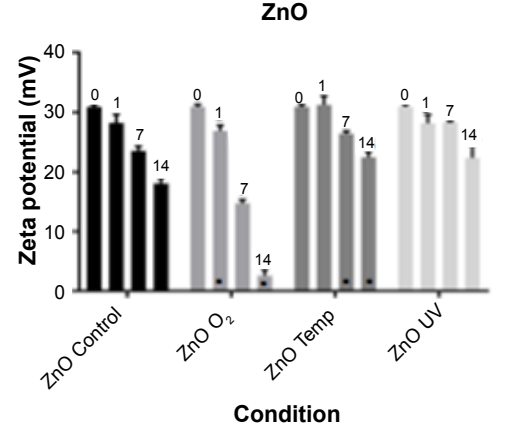

$\mathrm{TiO}_{2}$

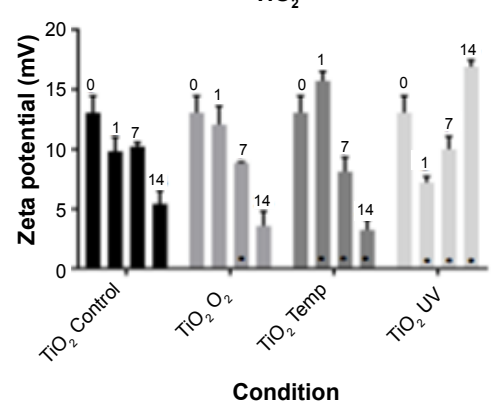

$\mathrm{TiO}_{2}$ Control

$=\mathrm{TiO}_{2} \mathrm{O}_{2}$

= $\mathrm{TiO}_{2}$ Temp

- $\mathrm{TiO}_{2} \mathrm{UV}$

•" Statistically Significan 
C)
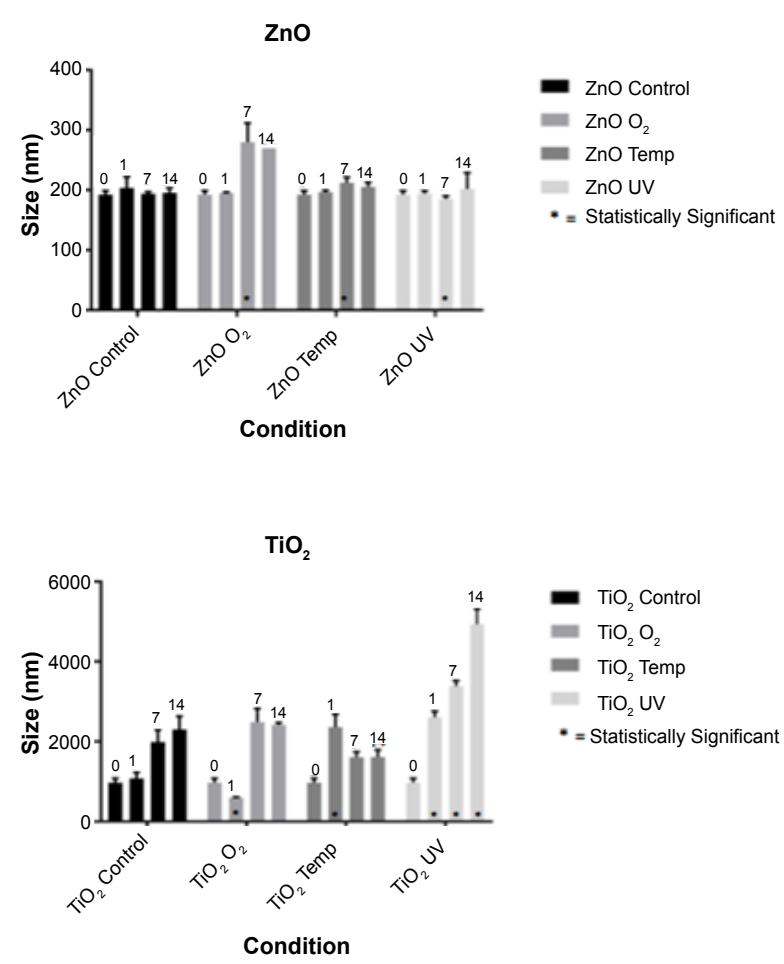

GO

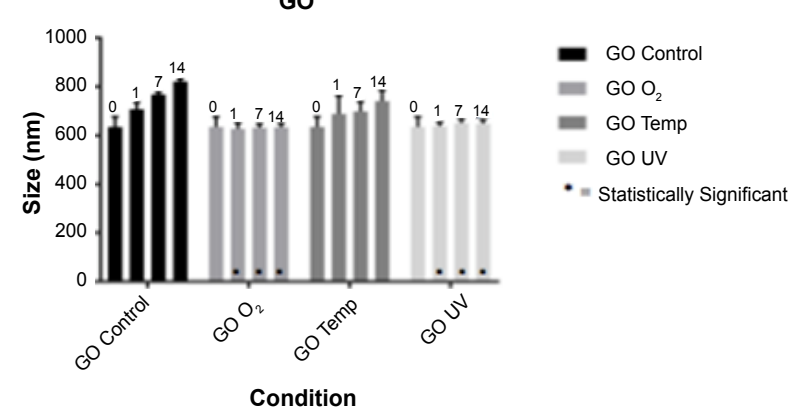

ZnO NW

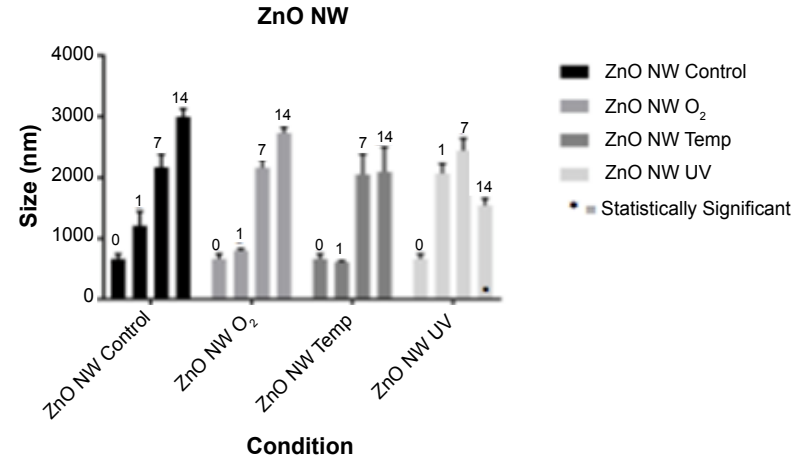

Figure 1: Physicochemical properties of the selected nanomaterials exposed to environmental conditions: A) Absorbance; B) Zeta potential, and C) Particle size.

and UV-irradiation exposure over time.

The declines occurred especially at days 7 and 14 may suggest a longer transformation time for zinc-involved nanoparticles. A study involving $\mathrm{ZnO}$ showed that longer wavelengths of light are not absorbed well by $\mathrm{ZnO}$, but shorter wavelengths are [33], with a relative peak witnessed at $377 \mathrm{~nm}$ and attributed to $\mathrm{ZnO}$ in the organic phase. The UV and oxygenated conditions seem to be crucial in improving performance of devices that incorporate ZnO. For instance, a recent study revealed improved performance of a gas sensor because of increasing viability of using $\mathrm{ZnO}$ as a gas sensor when either an oxygen-rich or ultraviolet (UV) radiation environmental condition was given [23].

Slight absorbance decreases over time under the environmental exposure conditions were observed from $\mathrm{TiO}_{2}$ nanoparticles (NPs), indicating that $\mathrm{TiO}_{2}$ NPs do not experience large changes to optical sensitivity over 14 days, 56 accumulative hours, of environmental exposure conditions. The absorbance measurement was reported to be dependent on molar absorptivity, path length, and the concentration of the absorbing chemical species [34]. In addition, absorbance measured by UV-Vis Spectroscopy highlights the concentration of a material, in addition to the absorbance measurement [35]. Results suggest at a constant concentration of the model NMs, various factors including UV irradiation, temperature, and oxygenation influence the absorbance of a material. A study by Fleger and Rosenbluh [36], investigating the absorption spectra of gold and silver NPs, elucidated the influence of concentration of the material on the absorption spectra, with peaks ranging from 450 to $500 \mathrm{~nm}$.

Zeta potential values measured were also assessed as one of physicochemical properties of the model nanomaterials under the environmental exposure conditions. Figure $1 \mathrm{~B}$ exhibits changes of the zeta potential, which describes ion adsorption and electrostatic interactions between charged particles [37]. While measuring zeta potential values may be challenging because of the complex nature of NMs under the environmental exposure conditions, the values could still be used as a metric of stability. As shown in Figure 1B, overall decrease in zeta potential values was found regardless of the type of NMs under the environmental exposure 
conditions over time.

Among the NMs, significant decreases in zeta potential of $\mathrm{TiO}_{2}$ were noted under temperature, oxygenation, and UV irradiation. Semiconductor NPs such as $\mathrm{TiO}_{2}$ that act as photocatalysts are well known to undergo a chemical transformation from UV exposure in an aqueous solution, thus producing hydroxyl radicals and forming a redox condition $[38,39]$. It was shown that chemical transformation increases the hydrophilicity of a nanomaterial while reducing fouling and improving water flux [39]. This phenomenon was hypothesized to cause a decline in the zeta potential of $\mathrm{TiO}_{2} / \mathrm{GO}$ membranes that were exposed to UV radiation, which similar observation occurred in the present study. When NMs form hydroxyl groups during the hydrophilic conversion of $\mathrm{TiO}_{2} / \mathrm{GO}$ membranes from UV activation, zeta potential was decreased [39].

Similarly, GO displayed a statistically significant decline in zeta potential from all exposure conditions. Research has shown possible reduction of GO by UV radiation in the presence of photocatalysts and reducing agents $[39,40]$; however, the sole effect of UV radiation without reducing agents is not well known [41]. To the best of our knowledge, there is a lack of research in the effects of temperature and oxygenation on the zeta potential of GO NPs.

Additionally, our results showed that $\mathrm{ZnO}$ and ZnO NW experienced a decline in zeta potential under the environmental exposure conditions. These results suggest instability (destabilization) and resultant aggregation of aged $\mathrm{ZnO} / \mathrm{ZnO} \mathrm{NW}$ and transformed $\mathrm{ZnO} / \mathrm{ZnO} \mathrm{NW}$ under oxygenation, temperature change, and UV irradiation. A recent study [42], which investigated the effect of increasing temperature on zeta potentials of $\mathrm{NaCl}$ and $\mathrm{MgCl}_{2}$ electrolytes, revealed a decrease in the magnitude of zeta potentials (it becomes less negative) as temperature increases.

By contrast, another study [43] showed a more negative zeta potential value from $\alpha-\mathrm{Al}_{2} \mathrm{O}_{3}$ /aqueous solution with $10 \mathrm{mgL}^{-1}$ fluoride ions added when temperature increased, possibly due to fluoride ions that chemisorb onto the solution. A change in $\mathrm{pH}$ was measured and indicated to remain $\mathrm{pH}$ around $7.5( \pm 5)$, indicating no remarkable change in $\mathrm{pH}$ and no influencing $\mathrm{pH}$ factor on each environmental exposure condition.
Particle sizes are related to stability and may affect the sensitivity and selectivity of NMs being used in chemical nanosensors. Hence, particle sizes of the model NMs were examined under the environmental exposure conditions. As shown in Figure 1C, significant particle size increase was observed from $\mathrm{TiO}_{2}$ and $\mathrm{ZnO} \mathrm{NW}$ under the exposure conditions over time, whereas a particle size increase of $\mathrm{ZnO}$ under oxygenation and a slight particle size increase in GO under the aged condition were observed. Particularly, particle sizes of GO remained few deviations under both oxygenation and ultraviolet conditions, although slightly increasing particle sizes of aged GO over time were found. Several studies [44-47] have shown that hydrophobic graphene sheets form irreversible aggregates through Van der Waals interactions. The oxidation of graphene to graphene oxide was reported to introduce hydroxyl and epoxide functional groups to the basal planes and carbonyl and carboxyl groups to the sheet edges, thus making GO sheets highly hydrophilic, thereby allowing dispersion in water and reducing aggregation [44-47].

Such dispersion of GO colloids is further stabilized by the highly negative charge of these colloids that causes electrostatic repulsions [44]. While GO tends to form less aggregation in water compared to graphene itself because of the hydrophilic nature of $\mathrm{GO}$, the increase of particle size in the aged GO over time (Figure 1C) is likely to be attributed to the decrease in surface charge seen in the zeta potential. This in turn, would decrease the electrostatic repulsions between the GO colloids and lead to an increase in aggregation of these colloids, increasing the particle size measurements over time. Several studies have reported reduction in the particle size of GO when GO undergoes photoreduction reactions $[48,49]$. The photoreduction reactions significantly decrease the oxygen-containing functional groups (mostly epoxy and carbonyl groups) under UV irradiation, thereby producing defects in the basal planes of $\mathrm{GO}$ and forming holes and breakage of the nanosheets $[48,49]$. These studies are consistent with the observation in $\mathrm{GO}$ samples exposed to UV irradiation (Figure 1C).

In contrast to little changes in particle sizes of $\mathrm{GO}$, a significant increase in particle size of the $\mathrm{TiO}_{2}$ nanoparticles after long-term UV irradiation was observed. These results are consistent with other 

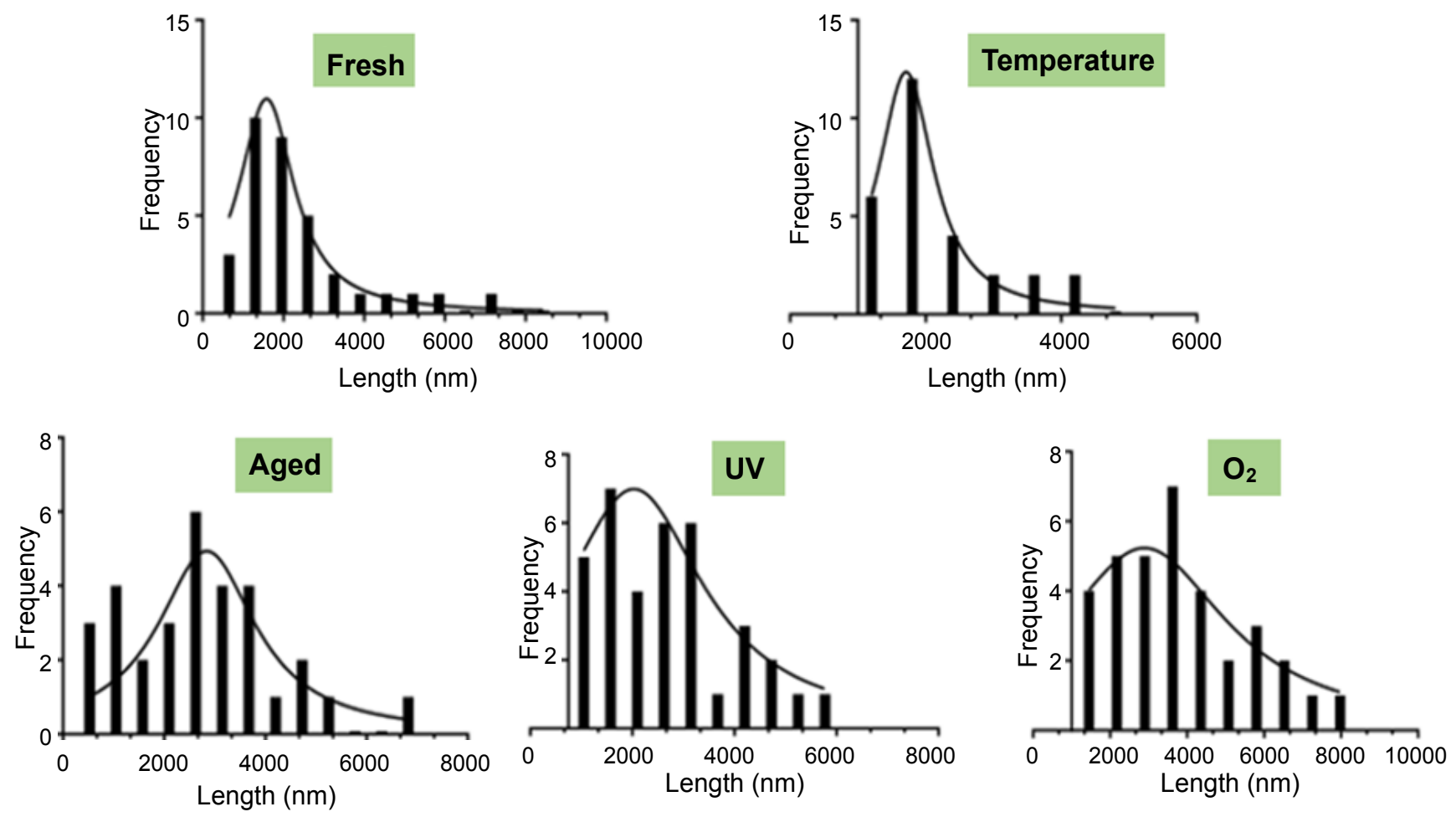

Figure 2: TEM size distribution (length) of CNTs exposed to environmental conditions.

studies $[50,51]$ in which UV irradiation has shown aggregation of $\mathrm{TiO}_{2}$ particles as a consequence of surface interactions. Increased $\mathrm{TiO}_{2}$ aggregation after UV irradiation is caused by the reduction in surface charge from dominant attractive Van der Waals forces over electrostatic repulsive forces $[50,51]$. Slight deviations in particle sizes of $\mathrm{ZnO}$ were observed especially under oxygenation. The extent to which particle size increases seems to depend on the types of NMs and the environmental exposure conditions.

Measuring particle size and zeta potential of CNTs in the aqueous phase was impossible because of significant and instant aggregation that exceeds the maximum values of which the equipment (Malvern Zetasizer) can analyze and measure particle sizes and zeta potential. As shown in Figure 2, most CNT particles are distributed within 1,000-1,500 nm at maximum, whereas under the environmental exposure conditions (temperature, aging, UV irradiation, and oxygenation), the maximum particle sizes are widely distributed: The fresh CNTs are shifted towards larger size ranges mostly exceeding 2,000 $\mathrm{nm}$, indicating significant aggregation and possibly larger aggregation, especially under UV and oxygenation conditions.
Physical morphology changes were noted from the model NMs under the environmental exposure conditions, as shown in the SEM images (Figure 3). Most NMs showed significant aggregation. For instance, $\mathrm{TiO}_{2} \mathrm{NPs}$, except fresh $\mathrm{TiO}_{2}$ (control), revealed significant aggregation. Similarly, SEM images of GO NPs showed aggregates surrounding the GO surfaces under the environmental exposure conditions (aging, oxygenation, temperature, and UV irradiation), although the extent of aggregation was not significant compared to $\mathrm{TiO}_{2}$ SEM images. One of the NMs, particularly CNTs, exhibited significant aggregates even including fresh CNTs (no aging and environmental exposure conditions). Changes in shape were also found in $\mathrm{ZnO} N W$ and $\mathrm{ZnO}$ NPs. As shown in Figure 3, fresh $\mathrm{ZnO} \mathrm{NW}$ is needle-shaped, whereas the other treated samples (aging, $\mathrm{O}_{2}$, temperature, and UV) showed a plate shape, indicating morphology change possibly due to chemical reactions under the exposure conditions. Similarly, compared to fresh $\mathrm{ZnO}$ (no aging and environmental exposure conditions), all treated samples (under aging, $\mathrm{O}_{2}$, temperature, and UV irradiation exposure conditions) revealed aggregates to some extent. Notably, under oxygenation, the morphology of $\mathrm{ZnO}$ was changed from a round to a star shape. 

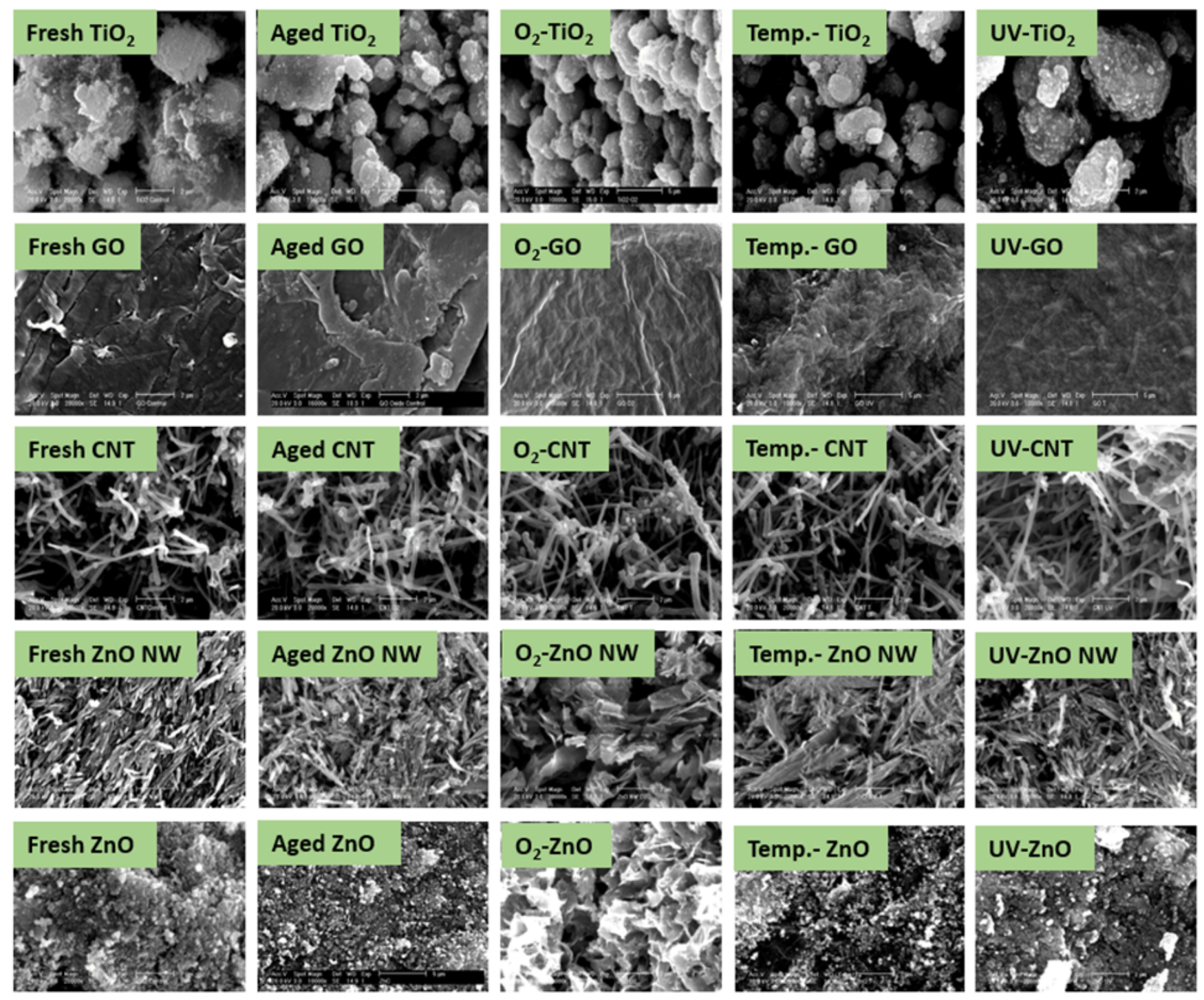

Figure 3: SEM images of the selected nanomaterials exposed to environmental conditions.

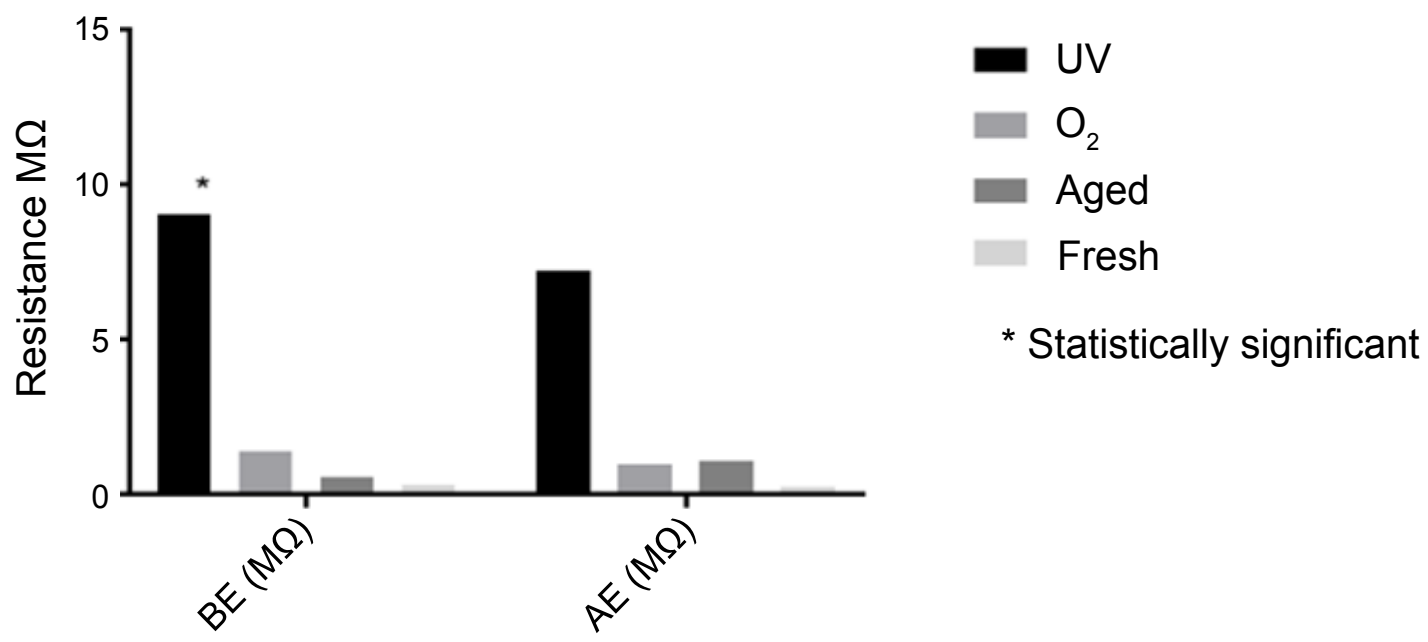

Figure 4: Electrical resistance of GO-CNT nanocomposite thin films before (BE) and after (AE) exposure to $\mathrm{CO}_{2}$ under environmental conditions. 


\section{Electrical resistance of GO-CNT thin films on environmental exposure conditions}

As observed in our study shown in Section 3.1, the extent of changes in physicochemical properties of NMs depends on not only different environmental conditions but also the types of NMs. These findings are further detailed in terms of electrical resistance of GO-CNT thin films after exposure to the environmental conditions at a constant concentration of GO-CNT nanocomposite films. Given the excellent property of CNTs and GO (i.e., hybrid gas sensors incorporating CNTs into GO because of increasing electrical conductance of CNTs or GO) $[2,16,18]$, the nanocomposite thin films consisting of GO-CNT were chosen and examined in terms of their electric resistance under UV irradiation, oxygenation, and aging after exposure to $\mathrm{CO}_{2}$ to assess sensitivity to detect trace levels of gas contaminants (Figure 4).

According to several studies [52-54], a change in resistance occurs when a chemical reaction is carried out within the surface of the chip whose resistance is being measured. It was noted that such a change of resistance occurred due to the depletion or accumulation of charge carriers $\left(\mathrm{CO}_{2}\right)[55,56]$. These results are consistent with the present study, in which oxygenated GO-CNT nanocomposite thin films showed a decrease in sensitivity after exposure to $\mathrm{CO}_{2}$. The UV-irradiated GO-CNT nanocomposite thin films also revealed a significant change in sensitivity before and after being exposed to $\mathrm{CO}_{2}$. These results indicate that a long-term exposure to either UV-irradiation or oxygen may cause the sensitivity (resistance) of GO-CNT nanocomposite thin films to decrease when exposed to a gas contaminant such as $\mathrm{CO}_{2}$.

Compared to exposure to either UV irradiation or oxygen, the aged and fresh GO-CNT nanocomposite thin films showed small changes in sensitivity from exposure to $\mathrm{CO}_{2}$, indicating that the interaction of the gas with the chip's surface does not change its sensitivity; thus, aged and fresh environmental conditions may not noticeably affect the electrical properties of the GO and CNT nanocomposite (Figure 4). Consequently, space environmental conditions may influence sensitivity and selectivity of chemical sensors in detecting gaseous contaminants, and the degree of changes may depend on physicochemical properties and types of NPs.

\section{Conclusions}

In our study, commonly used NMs in space products, including chemical sensors, were exposed to various environmental conditions to assess changes in physicochemical properties and the conditions' resultant influence on sensitivity and selectivity to detect trace levels of contaminants. Results indicated that although significant changes were noted in particle size, zeta potential, and absorbance when the selected $\mathrm{NMs}\left(\mathrm{TiO}_{2}, \mathrm{ZnO}, \mathrm{ZnO}\right.$ NW, CNTs, and GO) were exposed to UV irradiation and oxygenation, the degree of such changes depended on the type of NMs. For instance, the transformation of $\mathrm{ZnO}$ and $\mathrm{ZnO} \mathrm{NW}$ was apparent, as shown in significant declining trends on absorbance, while little changes were observed in absorbance and size from GO. A significant increase in particle size for all exposure conditions from $\mathrm{ZnO}$ $\mathrm{NW}$ and $\mathrm{TiO}_{2}$ - especially under $\mathrm{O}_{2}$, temperature (50 ${ }^{\circ} \mathrm{C}$ ), and UV-irradiation exposure conditions - was observed.

A declining trend for zeta potential over time from all types of NPs was found, indicating destabilization, followed by aggregation. NPs that act as photocatalysts, e.g., $\mathrm{TiO}_{2}$, may undergo a chemical transformation under UV exposure. As CNTs underwent aging and exposure to the environmental conditions, a wider range of size distribution occurred (beyond 2,000 nm), indicating agglomeration caused by aging and exposure conditions. Most NMs underwent significant instability with aggregation and morphology changes, especially from $\mathrm{ZnO}$ and $\mathrm{ZnO} \mathrm{NW}$, was observed as plateshaped ZnO NW from a needle-shaped one and a star-shaped $\mathrm{ZnO}$ from a round-shaped one. A study on electrical resistance due to exposure to a gaseous contaminant suggested that $\mathrm{CO}_{2}$-sensing ability of GO-CNT hybrid thin films is influenced by UV irradiation and oxygenation conditions; that is, after exposure to $\mathrm{CO}_{2}$, declines in electrical resistance were noted from UV-irradiated or oxygenated GOCNT thin films.

Nanomaterials, as routes for advancement to a wide array of conventional applications across many disciplines, are promising for gas-sensing applications, given that they can enhance early detection of gaseous contaminants because of their higher surface area and physicochemical reactivity. In space exploration, early detection of gaseous health hazards is a critical component of not only 
astronaut safety but also mission completion. The present study has explored how NMs are characterized in the perspective of size, electrical charge, absorbance, electrical resistance, and morphology changes when the NMs undergo transformation and/or aging processes under environmental exposure conditions. These findings, in turn, can assist in designing and developing new chemical sensors, thus better accommodating space applications.

\section{Acknowledgments}

This research was supported by the NASA through the University of Central Florida's NASA Florida Space Grant Consortium and Space Florida (Award: NNX15AI10H).

\section{References}

1. S Chopra, K McGuire, N Gothard, AM Rao (2003) Selective gas detection using a carbon nanotube sensor. Appl Phys Lett 83: 2280.

2. F Yavari, Z Chen, AV Thomas, W Ren, H-M Cheng, et al. (2011) High sensitivity gas detection using a macroscopic three-dimensional graphene foam network. Scientific Reports 1.

3. G Lu, LE Ocola, J Chen (2009) Reduced graphene oxide for room-temperature gas sensors. Nanotechnology 20: 445502.

4. S Manzetti, D Vasilache, E Francesco (2015) Emerging carbon-based nanosensor devices: Structures, functions and applications. Advances in Manufacturing 3: 63-72.

5. O Gohardani, MC Elola, C Elizetxea (2014) Potential and prospective implementation of carbon nanotubes on next generation aircraft and space vehicles: A review of current and expected applications in aerospace sciences. Progress in Aerospace Sciences 70: 42-68.

6. H Kataura, Y Kumazawa, Y Maniwa, I Umezu, S Suzuki, et al. (1999) Optical properties of single-wall carbon nanotubes. Synthetic Metals 103: 2555-2558.

7. M Maruthupandy, Y Zuo, J-S Chen, J-M Song, H-L Niu, et al. (2017) Synthesis of metal oxide nanoparticles ( $\mathrm{CuO}$ and $\mathrm{ZnO} \mathrm{NPs}$ ) via biological template and their optical sensor applications. Applied Surface Science 397: 167-174.

8. P Falcaro, R Ricco, A Yazdi, I Imaz, S Furukawa, et al. (2016) Application of metal and metal oxide nanoparticles@MOFs. Coordination Chemistry Reviews 307: 237-254.

9. S Basu, P Bhattacharyya (2012) Recent developments on graphene and graphene oxide based solid state gas sensors. Sensors and Actuators B: Chemical 173: 1-21.

10.MP McGrath, A Pham (2005) Microwave vertically aligned carbon nanotube array sensors for ammonia detection. IEEE Sensor.

11.Committee on Spacecraft Exposure (2008) Spacecraft maximum allowable concentrations for selected airborne contaminants. National Academies Press 5.

12.NJ Langford (2005) Carbon dioxide poisoning. Toxicol Rev 24: 229-235.

13.RA Vaia (2012) Nanomaterials and future aerospace technologies: Opportunities and challenges. Proceedings of SPIE - The International Society for Optical Engineering 8373.

14.AM Beese, D Papkov, SLi, Y Dzenis, HD Espinosa (2013) In situ transmission electron microscope tensile testing reveals structure-property relationships in carbon nanofibers. Carbon 60: 246-253.

15.W Kong, X Yang, M Yang, H Zhou, Z Ouyang, et al. (2016) Photoluminescent nanosensors capped with quantum dots for high-throughput determination of trace contaminants: Strategies for enhancing analytical performance. TrAC Trends in Analytical Chemistry 78: 36-47.

16.R Ruoff (2008) Graphene: Calling all chemists. Nat Nanotechnol 3: 10-11.

17.RH Binder (2017) Optical properties of graphene. World Scientific Publishing Co. Pte. Ltd.

18.WC Huang, HJ Tsai, TC Lin, WC Weng, YC Chang, et al. (2018) Incorporation of carbon nanotube and graphene in ZnO Nanorods-based hydrogen gas sensor. Ceramics International 4: 12308-12314.

19.O Lupan, V Cretu, V Postica, M Ahmadi, BR Cuenya, et al. (2016) Silver-doped zinc oxide single nanowire multifunctional nanosensor with a significant enhancement in response. Sensors and Actuators B: Chemical 223: 893-903.

20.CO Chey, X Liu, H Alnoor, O Nur, M Willander (2014) Fast piezoresistive sensor and UV photodetector based on Mn-doped ZnO nanorods. Physica Status Solidi (RRL) Rapid Research Letters 9: 87-91.

21.S Youssef, J Podlecki, R Habchi, M Brouche, A Foucaran, et al. (2012) Innovative prototype of a zinc-oxide based optical gas sensor. Sensors and Actuators B: Chemical 173: 391-395.

22.VK Jayaraman, AM Alvarez, M de la LO Zmador (2015) A simple and cost-effective zinc oxide thin film sensor for propane gas detection. Materials Letters 157: 169-171. 
23. L Zhu, W Zeng (2017) Room-temperature gas sensing of ZnO-based gas sensor: A review. Sensors and Actuators A: Physical 267: 242-261.

24.KC Chiang, W-Y Wu, J-M Ting (2011) Enhanced lateral growth of zinc oxide nanowires on sensor chips. Journal of the American Ceramic Society 94: 713-716.

25.F Huber, S Riegert, M Madel, K Thonke (2017) $\mathrm{H}_{2} \mathrm{~S}$ Sensing in the ppb regime with zinc oxide nanowires. Sensors and Actuators B: Chemical 239: 358-363.

26.DS Dhawale, RR Salunkhe, UM Patil, KV Gurav, AM More, et al. (2008) Room temperature liquefied petroleum gas (LPG) sensor based on p-polyaniline/ $\mathrm{n}-\mathrm{TiO}_{2}$ heterojunction. Sensors and Actuators B: Chemical 134: 988-992.

27.SG Pawar, MA Chougule, S Sen, VB Patil (2012) Development of nanostructured polyaniline-titanium dioxide gas sensors for ammonia recognition. Journal of Applied Polymer Science 125: 1418-1424.

28.A Ghicov, P Schmuki (2009) Self-ordering electrochemistry: $A$ review on growth and functionality of $\mathrm{TiO}_{2}$ nanotubes and other selfaligned $\mathrm{MO}(\mathrm{x})$ structures. Chem Commun (Camb) 20: 2791-2808.

29.J Bai, B Zhou (2014) Titanium dioxide nanomaterials for sensor applications. Chemical Reviews 114: 10131-10176.

30.Z Ye, H Tai, R Guo, Z Yuan, C Liu, et al. (2017) Excellent ammonia sensing performance of gas sensor based on graphene/titanium dioxide hybrid with improved morphology. Applied Surface Science 419: 84-90.

31.C Hu, FE Muller-Karger, RG Zepp (2002) Absorbance, absorption coefficient, and apparent quantum yield: A comment on common ambiguity in the use of these optical concepts. American Society of Limnology and Oceanography 47: 1261-1267.

32. M Hasani, M Mahdavian, H Yari, B Ramezanzadeh (2018) Versatile protection of exterior coatings by the aid of graphene oxide nano-sheets; comparison with conventional UV absorbers. Progress in Organic Coatings 116: 90-101.

33.VE Podasca, TB Buruiana, EC Buruiana (2016) UVcured polymeric films containing $\mathrm{ZnO}$ and silver nanoparticles with UV-vis light-assisted photocataIytic activity. Applied Surface Science 377: 262-273.

34.JH Hardesty, B Attili (2010) Spectrophotometry and the Beer-Lambert Law: An important analytical technique in chemistry.

35. RSC (2009) Ultraviolet - Visible Spectroscopy (UV).
36.Y Fleger, M Rosenbluh (2009) Surface plasmons and surface enhanced Raman spectra of aggregated and alloyed gold-silver nanoparticles. Research Letters in Optics: 2009.

37.S Li, P Leroy, F Heberling, N Devau, D Jougnot, et al. (2011) Influence of surface conductivity on the apparent zeta potential of $\mathrm{TiO}_{2}$ nanoparticles. Journal of Colloid and Interface Science 356: 442-453.

38.MK Nowotny, J Nowotny (2010) Solid state chemistry and photocatalysis of titanium dioxide. Scientific Net Publisher 162: 360.

39.MLK Pedersen, TR Jensen, SV Kucheryavskiy, ME Simonsen (2018) Investigation of surface energy, wettability and zeta potential of titanium dioxide/ graphene oxide membranes. Journal of Photochemistry and Photobiology A: Chemistry 366: 162-170.

40.L Guardia, S Villar-Rodil, JI Paredes, R Rozada, A Martínez-Alonso, et al. (2012) UV light exposure of aqueous graphene oxide suspensions to promote their direct reduction, formation of graphene-metal nanoparticle hybrids and dye degradation. Carbon 50: 1014-1024.

41. YH Ding, P Zhang, Q Zhuo, HM Ren, ZM Yang, et al. (2011) A green approach to the synthesis of reduced graphene oxide nanosheets under UV irradiation. Nanotechnology 22: 205601.

42.J Vinogradov, MD Jackson, M Chamerois (2018) Zeta potential in sandpacks: Effect of temperature, electrolyte $\mathrm{pH}$, ionic strength and divalent cations. Colloids and Surfaces A: Physicochemical and Engineering Aspects 553: 259-271.

43.A López Valdivieso, JL Reyes Bahena, S Song, R Herrera Urbina (2006) Temperature effect on the zeta potential and fluoride adsorption at the $\alpha-\mathrm{Al}_{2} \mathrm{O}_{3} /$ aqueous solution interface. J Colloid Interface Sci 298: 1-5.

44.D Li, MB Muller, S Gilje, RB Kaner, GG Wallace (2008) Processable aqueous dispersions of graphene nanosheets. Nature Nanotechnology 3: 101-105.

45.S Stankovich, DA Dikin, GHB Dommett, KM Kohlhaas, EJ Zimney, et al. (2006) Graphene-based composite materials. Nature 442: 282-286.

46.A Lerf, H He, M Forster, J Klinowski (1998) Structure of graphite oxide revisited. J Phys Chem B 102: 44774482.

47.T Szabo, O Berkesi, P Forgo, K Josepovits, Y Sanakis, et al. (2006) Evolution of surface functional groups in a series of progressively oxidized graphite oxides. Chem Mater 18: 2740-2749. 
48.AS Adeleye, X Wang, F Wang, R Hao, W Song, et al. (2018) Photoreactivity of graphene oxide in aqueous system: Reactive oxygen species formation and bisphenol A degradation. Chemosphere 195: 344350.

49.Y Matsumoto, M Koinuma, S Ida, S Hayami, T Taniguchi, et al. (2011) Photoreaction of graphene oxide nanosheets in water. J Phys Chem 115: 1928019286.

50.J Sun, L-H Guo, H Zhang, L Zhao (2014) UV irradiation induced transformation of $\mathrm{TiO}_{2}$ nanoparticles in water: Aggregation and photoreactivity. Environ Sci Technol 48: 11962-11968.

51.C Zhou, Y Bashirzadeh, TA Bernadowski, Jr X Zhang (2016) UV light-induced aggregation of titania submicron particles. Micromachines (Basel) 7: 203.

52.J Guo, J Zhang, M Zhu, D Ju, H Xu, et al. (2014) Highperformance gas sensor based on $\mathrm{ZnO}$ nanowires functionalized by $\mathrm{Au}$ nanoparticles. Sensors and Actuators B: Chemical 199: 339-345.
53.M Yuasa, T Masaki, T Kida, K Shimanoe, N Yamazoe (2009) Nano-sized PdO loaded $\mathrm{SnO}_{2}$ nanoparticles by reverse micelle method for highly sensitive $\mathrm{CO}$ gas sensor. Sensors and Actuators B: Chemical 136: 99104.

54.T Kida, A Nishiyama, Z Hua, K Suematsu, M Yuasa, et al. (2014) $\mathrm{WO}_{3}$ nanolamella gas sensor: Porosity control using $\mathrm{SnO}_{2}$ nanoparticles for enhanced $\mathrm{NO}_{2}$ sensing. Langmuir 30: 2571-2579.

55.A Kaniyoor, $R$ Imran Jafri, $T$ Arockiadoss, $S$ Ramaprabhu (2009) Nanostructured Pt decorated graphene and multi walled carbon nanotube-based room temperature hydrogen gas sensor. Nanoscale 1: 382-386.

56.G Sakai, NS Baik, N Miura, N Yamazoe (2001) Gas sensing properties of tin oxide thin films fabricated from hydrothermally treated nanoparticles: Dependence of $\mathrm{CO}$ and $\mathrm{H}_{2}$ response on film thickness. Sensors and Actuators B: Chemical 77: 116-121.

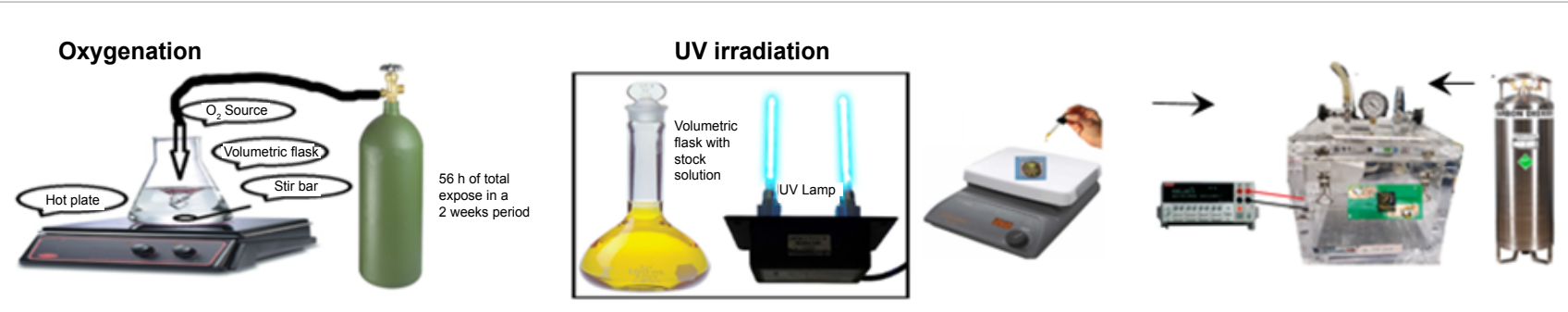

Scheme 1: Schematic diagram of electric resistance experiments with GO-CNT thin films. 